\title{
Contrast-enhanced whole-heart coronary MRA at 3.0T for the evaluation of cardiac venous anatomy
}

\author{
Heng Ma - Qing Tang • Qi Yang - Xiaoming Bi • Han Li \\ Lan Ge $\cdot$ Kai Lin $\cdot$ Dong Xu $\cdot$ Xiangying Du $\cdot$ Jie Lu $\cdot$ \\ Jing An $\cdot$ Lixin Jin $\cdot$ Renate Jerecic $\cdot$ Kuncheng Li $\cdot$ Debiao Li
}

Received: 26 August 2010/ Accepted: 19 November 2010/Published online: 1 December 2010

(C) The Author(s) 2010. This article is published with open access at Springerlink.com

\begin{abstract}
This study was designed to evaluate the value of contrast-enhanced whole-heart coronary MRA (CMRA) at 3.0T in depicting the cardiac venous anatomy. In cardiac resynchronization therapy (CRT), left ventricular (LV) pacing is achieved by positioning the LV lead in one of the tributaries of the coronary sinus (CS). Pre-implantation knowledge of the venous anatomy may help determine whether transvenous LV lead placement for CRT is feasible. Images of 51 subjects undergoing contrast-enhanced whole-heart CMRA at 3.0T were retrospectively analyzed. Data acquisition was performed using electrocardiographytriggered, navigator-gated, inversion-recovery prepared, segmented gradient-echo sequence. A 32-element cardiac coil was used for data acquisition. The visibility of
\end{abstract}

H. Ma · Q. Yang · X. Du · J. Lu · K. Li $(\bowtie)$

Department of Radiology, Xuanwu Hospital, Capital

Medical University, No. 45, Chang-Chun Street,

100053 Xuanwu District, Beijing, China

e-mail: likuncheng1955@yahoo.com.cn

Q. Tang · H. Li · D. Xu

Department of Cardiology, Xuanwu Hospital,

Capital Medical University, Beijing, China

X. Bi

Cardiovascular MR R\&D, Siemens Healthcare, Chicago,

IL, USA

L. Ge $\cdot$ K. Lin $\cdot$ D. Li

Department of Radiology, Northwestern University,

Suite 1600, 737 N. Michigan Ave.,

Chicago, IL 60611, USA the cardiac veins was graded visually using a 4-point scale (1: poor-4: excellent). The paired Student $t$ test was used to evaluate differences in diameters of the ostium of the CS in anteroposterior and superoinferior direction. The cardiac veins were finally evaluated in 48 subjects with three anatomic variations. The diameter of the CS ostium in the superoinferior direction $(1.13 \pm 0.26 \mathrm{~cm})$ was larger than in the anteroposterior direction $(0.82 \pm 0.19 \mathrm{~cm})(P<0.05)$. The mean visibility score of CS, posterior interventricular vein, posterior vein of the left ventricle, left marginal vein, and anterior interventricular vein was $4.0 \pm 0.0,3.4 \pm 0.5,3.4 \pm 0.5,3.0 \pm 0.8$, and $3.3 \pm$ 0.5 , respectively. In conclusion, contrast-enhanced whole-heart CMRA at 3.0T can depict the normal and variant cardiac venous anatomy.

J. An

Siemens Healthcare, MR Collaboration NE Asia,

Siemens Mindit Magnetic Resonance, Shenzhen, China

L. Jin · R. Jerecic

Siemens Healthcare, MR Collaboration NE Asia,

Siemens Limited China, Shanghai, China

D. $\mathrm{Li}$

Cedars-Sinai Medical Center and UCLA, Los Angeles, CA, USA 
Keywords Coronary MRA $\cdot$ Cardiac veins $\cdot 3.0 \mathrm{~T}$

\section{Introduction}

In patients with congestive heart failure, pacemaker leads for cardiac resynchronization therapy (CRT) are placed in one of the tributaries of the coronary sinus (CS) system, located in the lateral wall of the left ventricle [1]. Because the cardiac veins are variable in number, caliber, and course, pre-implantation knowledge of the venous anatomy may help cardiologists to decide whether intravenous implementation should be performed and choose the optimal placement of the left ventricular lead implantation. Thus, there is a clinical need for imaging the cardiac venous system.

There are only a few methods of imaging the cardiac venous system. Retrograde cardiac venography via the CS is the current gold standard for defining the cardiac venous anatomy. However, such a procedure is invasive, technically challenging, and unable to outline the cardiac arteriovenous relationship. Ideally, venous anatomy should be assessed before implantation noninvasively in the outpatient clinic to determine whether a transvenous approach is feasible. Therefore, efforts have been made over recent years to explore new methods of imaging. For example, multislice spiral computed tomography (MSCT) has become an important tool for noninvasive visualization of the cardiac venous system [2-5]. Recent studies using whole-heart steady-state free precession (SSFP) coronary MRA (CMRA) demonstrates that MR can visualize the anatomy of the cardiac venous system at 1.5T [6-10].

Contrast-enhanced whole-heart CMRA has been used to evaluate the coronary arteries at 3.0T [1113]. However, no data are currently available on the use of this 3.0T whole-heart CMRA technique to visualize the cardiac venous anatomy. The purpose of the work was to evaluate the value of contrastenhanced whole-heart CMRA at 3.0T in depicting the cardiac venous anatomy: the CS and its tributaries.

\section{Materials and Methods}

Study population

The anatomy of the cardiac veins was retrospectively studied in 51 consecutive subjects (45 patients with suspected coronary artery disease and 6 healthy volunteers; 26 men; age $59 \pm 11$ years) in whom 3.0T contrast-enhanced whole-heart CMRA was performed for non-invasive evaluation of the coronary arteries. The study was approved by the institutional review board and informed consents were obtained from each subject.

\section{Contrast-enhanced whole-heart CMRA}

The CMRA was performed on a 3.0T whole-body clinical scanner (MAGNETOM Tim Trio, Siemens Healthcare, Erlangen, Germany) equipped with a 32-element cardiac coil.

All images of coronary vessels were acquired during free-breathing with the subjects in a supine position. A navigator-gated, electrocardiography-triggered, fat saturated, segmented 3D fast low-angle shot (FLASH) sequence was utilized for CMRA [11]. Navigator pulses localized at the dome of the right hemidiaphragm with a 5-mm acceptance window was used for respiratory gating. The $3 \mathrm{D} \mathrm{k}$-space data were collected with a centric ordering scheme in the phaseencoding direction and a linear ordering scheme in the partition-encoding direction. A nonselective inversion pulse (TI $=200 \mathrm{~ms}$ ) was applied prior to the navigator-echo pulses to suppress the background signal. Sixty-four to 72 slices were acquired and interpolated to $128-144$ slices of $0.65 \mathrm{~mm}$ thick. To reduce the image acquisition time, parallel data acquisition was used in the phase-encoding direction with an acceleration factor of 3. Prior to coronary MRA, a cine scan was performed to identify cardiac phase with minimal motion of the right coronary artery (RCA) [11-13]. The data acquisition window and the number of $\mathrm{k}$ space lines acquired per heartbeat were set accordingly to synchronize data acquisition to minimal motion phases, either during systole or diastole. Other imaging parameters were as follows: $\mathrm{TR}=3.56 \mathrm{~ms}$, $\mathrm{TE}$ $=1.61 \mathrm{~ms}$, flip angle $=20^{\circ}$, number of lines per heartbeat $=30-50$, readout bandwidth $=709 \mathrm{~Hz} /$ pixel, and voxel size $=1.1 \times 1.1 \times 1.3 \mathrm{~mm}^{3}$ interpolated to $0.55 \times 0.55 \times 0.65 \mathrm{~mm}^{3}$.

$0.15 \mathrm{mmol} / \mathrm{kg}$ body weight of MultiHance (Bracco Imaging SpA, Milan, Italy) was slowly injected using a power injector (Spectris, Medrad, Indianola, PA, USA) at a rate of $0.3 \mathrm{ml} / \mathrm{s}$, immediately followed by $20 \mathrm{ml}$ of saline injected at the same rate. Imaging acquisition started $80 \mathrm{~s}$ after the initialization of contrast agent administration [11-13]. 
Data analysis

Data reformation and analysis were performed on a commercially available workstation (Leonardo, Siemens Healthcare, Erlangen, Germany).

\section{Anatomic observation}

The tributaries of the cardiac veins were identified on volume-rendered reconstructions. Thereafter, the course of the veins was evaluated in 3 orthogonal planes using multiplanar reformatting. The presence of the following cardiac veins was evaluated: CS, posterior interventricular vein (PIV), posterior vein of the left ventricle (PVLV), left marginal vein (LMV), and anterior interventricular vein (AIV) (Fig. 1).

\section{Quantitative data}

The ostium of the CS was defined as the site where the CS makes an angle with the right atrium (RA) in the crux cordis area [4, 5, 9]. Multiplanar reformatting was used to determine the size of the ostium of the CS in anteroposterior and superoinferior direction (Fig. 2) and to measure the starting diameter of each identified tributary. The length of the tributaries, the distance from the ostium to tributaries and the angle between the tributaries and CS or great cardiac vein were measured on volume-rendered reconstructions (Fig. 3). The angle of the CS ostium was measured in the axial images [14]. The visibility of the cardiac veins was graded visually using a 4-point scale by assessing raw images (1: poor, 2: moderate, 3: good, and 4: excellent) $[13,15]$.
Statistical analysis

A statistical software program, SPSS 13.0 (SPSS Inc., Chicago, IL, USA), was used for statistical analysis. Continuous variables were expressed as mean \pm standard deviation. Categorical variables were expressed as absolute number (percentage). The paired Student $t$ test was used to evaluate differences in diameters of the ostium of the CS in anteroposterior and superoinferior direction. Statistical tests were 2 -tailed, and a $P$ value $<0.05$ was considered to be significant.

\section{Results}

Acquisition time of whole-heart CMRA procedure was $7.1 \pm 2.2 \mathrm{~min}$. The CMRA was acquired during diastole in 43 subjects (acquisition window $139 \pm$ $41 \mathrm{~ms}$ ) and during systole in 8 subjects (acquisition window $91 \pm 9 \mathrm{~ms}$ ). The average navigator efficiency was $36 \%$.

The cardiac veins were finally evaluated in 48 of 51 subjects. Data from 3 subjects were excluded for analysis due to non-assessable image quality caused by poor contrast-to-noise ratio $(\mathrm{CNR})(\mathrm{n}=2)$ and motion artifacts $(\mathrm{n}=1)$.

\section{Anatomic observation}

The CS and PIV were observed in 48/48 (100\%) subjects. The PVLV was visualized in $42 / 48$ subjects $(88 \%)$, the LMV in 33/48 (69\%), and the AIV in 38/48 (79\%).

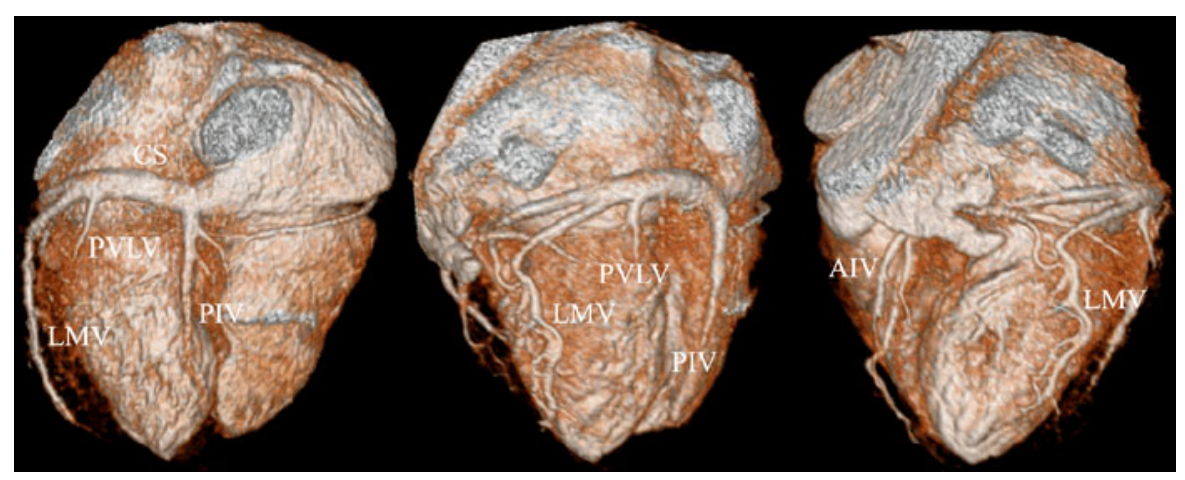

Fig. 1 Volume-rendered image provides an overview of cardiac venous anatomy and clearly depicts the coronary sinus (CS), posterior interventricular vein (PIV), posterior vein of the left ventricle (PVLV), left marginal vein (LMV), and anterior interventricular vein (AIV) 

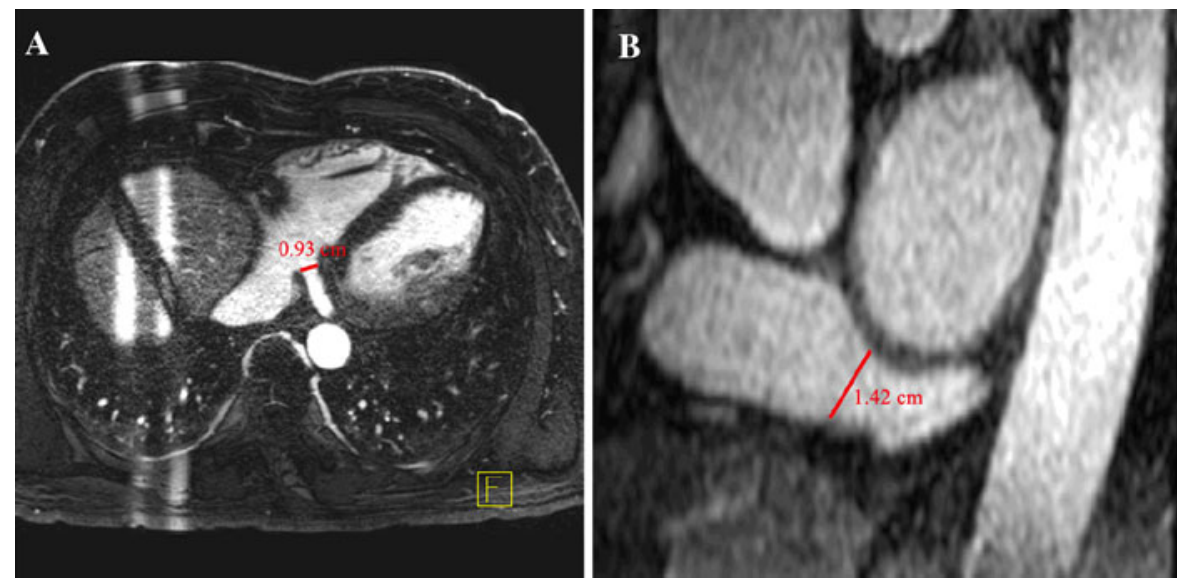

Fig. 2 a The CS ostium measured in the anteroposterior direction; transverse plane; b The CS ostium in superoinferior direction; coronary plane

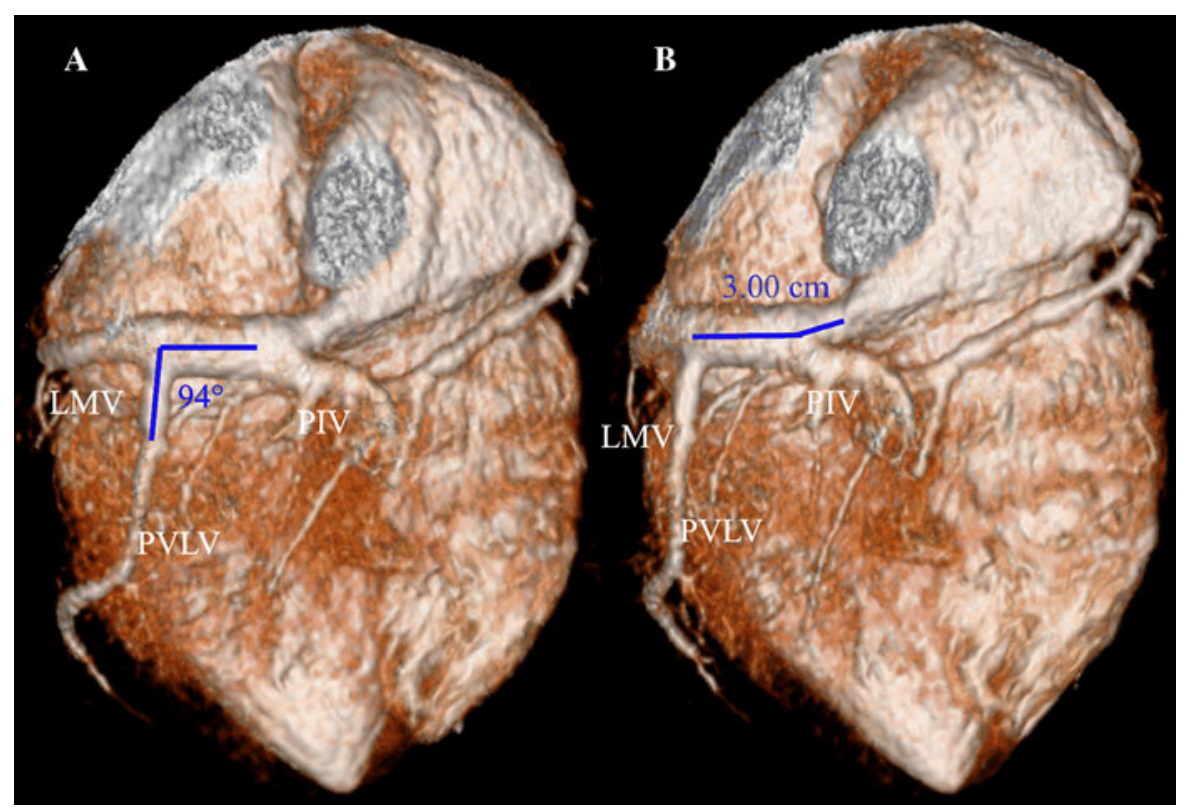

Fig. 3 Example of measurement of the angle between the tributaries and CS or great cardiac vein and of the distance from the ostium to tributaries

Anatomic variation

Two subjects showed common origin of PIV and PVLV from CS (Fig. 4a). In 1 subject, the small cardiac vein (SCV) connected to the PIV and the PIV connected to the CS at the crux cordis (Fig. 4b). This kind of anatomic variation was not included in Jongbloed's classification of variable anatomy (In Jongbloed's Variant 1: Continuity of the cardiac veins at the crux cordis. The SCV connects to the CS at the crux cordis) [4].

\section{Quantitative data}

Table 1 lists the quantitative data of the PIV, PVLV, LMV, and AIV. The diameter of the CS ostium in the superoinferior direction $(1.13 \pm 0.26 \mathrm{~cm})$ was larger than in the anteroposterior direction $(0.82 \pm 0.19 \mathrm{~cm})$ 


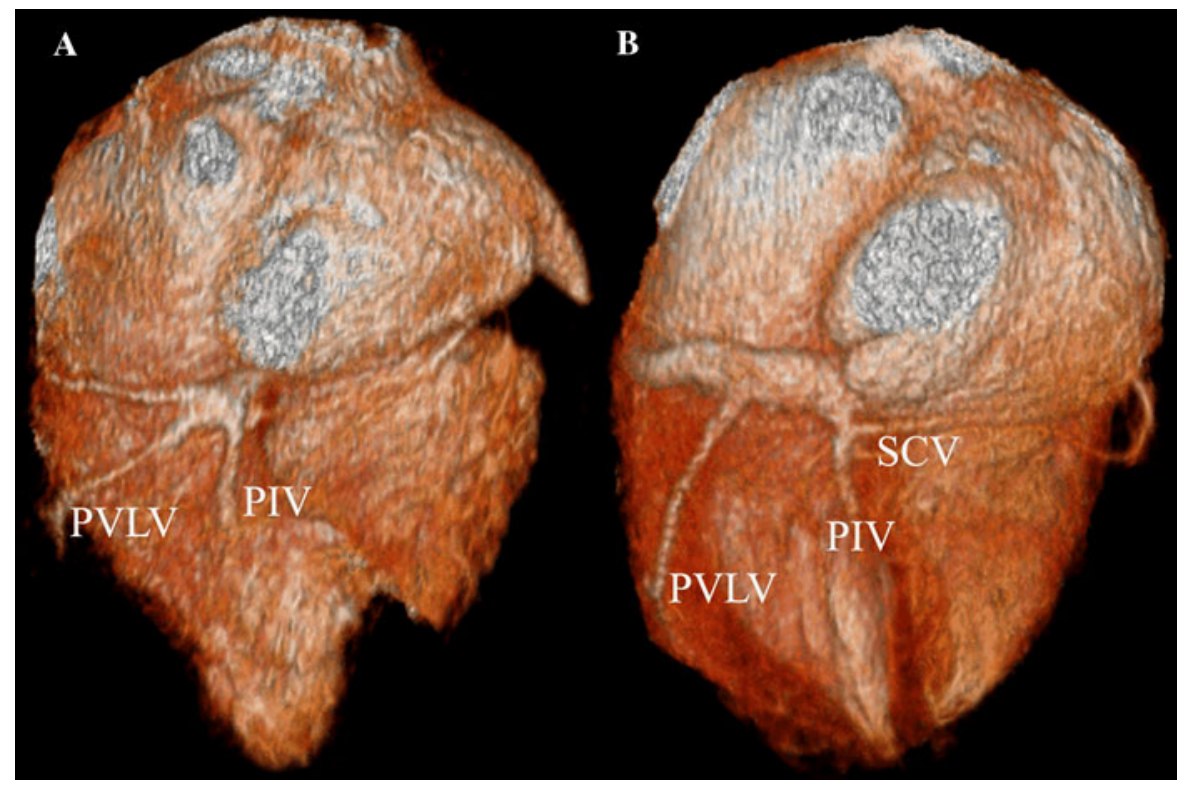

Fig. 4 a Volume-rendered image shows common origin of PIV and PVLV from the CS. b A new found anatomic variation: the small cardiac vein (SCV) connected to the PIV and the PIV connected to the CS at the crux cordis

Table 1 Quantitative measurement of cardiac veins from 48 subjects

\begin{tabular}{|c|c|c|c|c|}
\hline & $\begin{array}{l}\text { Ostial } \\
\text { diameter }(\mathrm{cm})\end{array}$ & Length $(\mathrm{cm})$ & $\begin{array}{l}\text { Distance from the } \\
\text { ostium of CS }(\mathrm{cm})\end{array}$ & $\begin{array}{l}\text { Angle between } \\
\text { the identified } \\
\text { veins and CS or } \\
\text { great cardiac vein }\end{array}$ \\
\hline PIV $(n=48)$ & $0.4 \pm 0.1$ & $3.1 \pm 1.8$ & $0.6 \pm 0.4$ & $81^{\circ} \pm 19^{\circ}$ \\
\hline PVLV $(n=42)$ & $0.3 \pm 0.1$ & $2.8 \pm 2.5$ & $3.0 \pm 1.0$ & $108^{\circ} \pm 26^{\circ}$ \\
\hline $\operatorname{LMV}(\mathrm{n}=33)$ & $0.2 \pm 0.1$ & $2.1 \pm 2.0$ & $6.5 \pm 1.1$ & $119^{\circ} \pm 30^{\circ}$ \\
\hline $\operatorname{AIV}(\mathrm{n}=38)$ & $0.3 \pm 0.1$ & $4.5 \pm 1.6$ & $9.9 \pm 1.5$ & $132^{\circ} \pm 17^{\circ}$ \\
\hline
\end{tabular}

Data are means \pm standard deviations

$A I V$ anterior interventricular vein, $C S$ coronary sinus, $L M V$ left marginal vein, $P I V$ posterior interventricular vein, $P V L V$ posterior vein of the left ventricle

$(P<0.05)$. The angle of the CS ostium was $59^{\circ} \pm 7^{\circ}$. The visibility is displayed in Table 2.

\section{Discussion}

This work shows that contrast-enhanced whole-heart CMRA at 3.0T can depict the normal and variant cardiac venous anatomy. Previous studies using navigator-gated, whole-heart SSFP CMRA demonstrate that MR can visualize the anatomy of the cardiac venous system at $1.5 \mathrm{~T}$ [6-10]. 3.0T systems have higher signal-to-noise ratio (SNR) and CNR than 1.5T [16-19]. Nevertheless, the SSFP imaging technique that has gained wide acceptance at $1.5 \mathrm{~T}$ is prone to imaging artifacts at $3.0 \mathrm{~T}$ because of the increased magnetic field inhomogeneity and radiofrequency (RF) distortion at higher field strengths. Compared to SSFP, spoiled gradient-echo imaging (e.g., FLASH) is less sensitive to static and RF field inhomogeneities, and results in more consistent image quality among subjects at 3.0T. The 3.0T imaging and contrast-enhancement combined with inversion-recovery preparation allow high contrast between blood and background tissue. The image quality of cardiac veins can be improved as a result. 
Table 2 Distribution of visibility grades of the cardiac veins

\begin{tabular}{llllll}
\hline \multicolumn{5}{l}{ Visibility grade $\mathrm{n}(\%)$} \\
\cline { 2 - 6 } & 1 & 2 & 3 & 4 & Mean \\
\hline CS & $0(0)$ & $0(0)$ & $0(0)$ & $48(100)$ & $4.0 \pm 0.0$ \\
PIV & $0(0)$ & $0(0)$ & $27(56)$ & $21(44)$ & $3.4 \pm 0.5$ \\
PVLV & $0(0)$ & $0(0)$ & $26(62)$ & $16(38)$ & $3.4 \pm 0.5$ \\
LMV & $2(6)$ & $5(15)$ & $18(55)$ & $8(24)$ & $3.0 \pm 0.8$ \\
AIV & $0(0)$ & $0(0)$ & $27(71)$ & $11(29)$ & $3.3 \pm 0.5$ \\
\hline
\end{tabular}

Data are $\mathrm{n}(\%)$ or means \pm standard deviations. The visibility grade of the cardiac veins: $1=$ poor; $2=$ moderate; 3 = good; 4 = excellent

$A I V$ anterior interventricular vein, $C S$ coronary sinus, $L M V$ left marginal vein, $P I V$ posterior interventricular vein, $P V L V$ posterior vein of the left ventricle

Currently, one of the major challenges for wholeheart CMRA is the long scan time and image artifacts caused by motion instability during the long scan time. Previous contrast-enhanced CMRA study at 3.0T using 12-channel coils [13] had reported reduced acquisition time compared to conventional SSFP CMRA at 1.5 T [15] (9 min vs. $13 \mathrm{~min}$ ). Using higher parallel imaging factor, the acquisition time is shortened to $7.1 \pm 2.2 \mathrm{~min}$ in this study. Sufficient SNR and image quality were maintained by imaging at 3.0T and utilization of 32-channel phased-array coils [20-22]. The shorter scan time has potential to improve spatial resolution and reduce image artifacts caused by increased motion instability during the long scan time [13, 15].

The results of our study confirm a substantial variation in the cardiac venous anatomy. First, the CS was analyzed. The finding that the CS ostium is ovally shaped agrees with observations in other cardiac veins $[4,5,9]$. Second, the tributaries of the CS were evaluated. Meisel et al. [23] studied 129 patients referred for cardioverter-defibrillator implantation with invasive venography and noted a PVLV in $55 \%$ and a LMV in $83 \%$. In studies using 16-slice or 64-slice MSCT, the prevalence of the PIV varied between 99 and 100\%, the prevalence of the PVLV between 82 and $96 \%$, and the prevalence of the LMV between 27 and $71 \%$ [4, 5]. Chiribiri et al. [9] retrospectively evaluated the feasibility of contrastenhanced SSFP CMRA at 1.5T to depict the anatomy of the cardiac venous system in 23 subjects and found the CS in $100 \%$ of subjects, the PIV in $96 \%$, the
PVLV in $78 \%$, the LMV in $70 \%$, and the AIV in $65 \%$. In our study using contrast-enhanced wholeheart CMRA at 3.0T in 48 subjects, the CS and PIV were observed in $100 \%$ of subjects, the PVLV in $88 \%$, the LMV in $69 \%$, and the AIV in $79 \%$.

Compared with other techniques for imaging the cardiac venous system, CMRA is noninvasive and does not require injection of iodinated contrast medium or expose patients to ionizing radiation. Thus, CMRA offers a relatively safe tool for the evaluation of the cardiac venous anatomy.

There were several limitations to this study. First, the CMRA used in this study was designed to provide optimal visualization of the coronary arteries, and may therefore be suboptimal for demonstration of the cardiac veins. Further studies will be required to define the best methodology to depict the cardiac venous anatomy by CMRA. Second, like previous 1.5T MR cardiac vein imaging studies [6-10], we did not compare our 3.0T whole-heart CMRA technique with the current invasive gold standard, retrograde cardiac venography. This issue is relevant because it is unknown whether cardiac vein branches not visualized were not present because of venous anatomic variation or present but not visualized. Third, the efficacy of CMRA for cardiac venous assessment specifically in patients with congestive heart failure has not yet been assessed and may prove more challenging. Finally, use of contrast media results in additional study cost as well as potential side effects. Additional precautions are necessary to rule out patients with poor renal function. It is also difficult to repeat the scan in the same imaging session if the acquisition is aborted or image quality is suboptimal [13]. Utilization of intravascular contrast agent may allow for a longer time window for contrast-enhanced imaging, as well as repeat of measurement if necessary.

In conclusion, contrast-enhanced whole-heart CMRA at 3.0T can depict the normal and variant cardiac venous anatomy. Pre-implantation knowledge of the venous anatomy may help determine whether transvenous left ventricular lead placement for CRT is feasible.

Acknowledgments This work was supported by National Institute of Health (NIBIB EB002623), National Natural Science Foundation of China (30828009), Siemens Medical Solutions, Bracco Diagnostics, and Invivo. 
Open Access This article is distributed under the terms of the Creative Commons Attribution Noncommercial License which permits any noncommercial use, distribution, and reproduction in any medium, provided the original author(s) and source are credited.

\section{References}

1. Kadish A, Mehra M (2005) Heart failure devices: implantable cardioverter-defibrillators and biventricular pacing therapy. Circulation 111:3327-3335

2. Knackstedt C, Mühlenbruch G, Mischke K, Schimpf T, Spüntrup E, Günther RW, Sanli B, Kelm M, Schauerte P, Mahnken AH (2008) Imaging of the coronary venous system in patients with congestive heart failure: comparison of 16 slice MSCT and retrograde coronary sinus venography: comparative imaging of coronary venous system. Int J Cardiovasc Imaging 24:783-791

3. Mlynarski R, Sosnowski M, Wlodyka A, Chromik K, Kargul W, Tendera M (2009) Optimal image reconstruction intervals for noninvasive visualization of the cardiac venous system with a 64-slice computed tomography. Int J Cardiovasc Imaging 25:635-641

4. Jongbloed MR, Lamb HJ, Bax JJ, Schuijf JD, de Roos A, van der Wall EE, Schalij MJ (2005) Noninvasive visualization of the cardiac venous system using multislice computed tomography. J Am Coll Cardiol 45:749-753

5. Van de Veire NR, Schuijf JD, De Sutter J, Devos D, Bleeker GB, de Roos A, van der Wall EE, Schalij MJ, Bax JJ (2006) Non-invasive visualization of the cardiac venous system in coronary artery disease patients using 64-slice computed tomography. J Am Coll Cardiol 48: 1832-1838

6. Younger JF, Plein S, Crean A, Ball SG, Greenwood JP (2009) Visualization of coronary venous anatomy by cardiovascular magnetic resonance. J Cardiovasc Magn Reson 11:26

7. Nezafat R, Han Y, Peters DC, Herzka DA, Wylie JV, Goddu B, Kissinger KK, Yeon SB, Zimetbaum PJ, Manning WJ (2007) Coronary magnetic resonance vein imaging: imaging contrast, sequence, and timing. Magn Reson Med 58:1196-1206

8. Rasche V, Binner L, Cavagna F, Hombach V, Kunze M, Spiess J, Stuber M, Merkle N (2007) Whole-heart coronary vein imaging: a comparison between non-contrast-agentand contrast-agent-enhanced visualization of the coronary venous system. Magn Reson Med 57:1019-1026

9. Chiribiri A, Kelle S, Götze S, Kriatselis C, Thouet T, Tangcharoen T, Paetsch I, Schnackenburg B, Fleck E, Nagel E (2008) Visualization of the cardiac venous system using cardiac magnetic resonance. Am J Cardiol 101: 407-412

10. Stoeck CT, Han Y, Peters DC, Hu P, Yeon SB, Kissinger KV, Goddu B, Goepfert L, Manning WJ, Kozerke S, Nezafat R (2009) Whole heart magnetization-prepared steady-state free precession coronary vein MRI. J Magn Reson Imaging 29:1293-1299
11. Bi X, Carr JC, Li D (2007) Whole-heart coronary magnetic resonance angiography at 3 Tesla in 5 minutes with slow infusion of Gd-BOPTA, a high-relaxivity clinical contrast agent. Magn Reson Med 58:1-7

12. Liu X, Bi X, Huang J, Jerecic R, Carr J, Li D (2008) Contrast-enhanced whole-heart coronary magnetic resonance angiography at $3.0 \mathrm{~T}$ : comparison with steady-state free precession technique at $1.5 \mathrm{~T}$. Invest Radiol 43: 663-668

13. Yang Q, Li K, Liu X, Bi X, Liu Z, An J, Zhang A, Jerecic R, Li D (2009) Contrast-enhanced whole-heart coronary magnetic resonance angiography at 3.0-T: a comparative study with X-ray angiography in a single center. J Am Coll Cardiol 54:69-76

14. Wijetunga M, Cuoco F, Ravi ND, Fuisz A, Strickberger SA (2006) Characterization of the coronary sinus ostium by cardiac magnetic resonance imaging. Am J Cardiol 98: $1400-1402$

15. Sakuma H, Ichikawa Y, Chino S, Hirano T, Makino K, Takeda K (2006) Detection of coronary artery stenosis with whole-heart coronary magnetic resonance angiography. J Am Coll Cardiol 48:1946-1950

16. Yang PC, Nguyen P, Shimakawa A, Brittain J, Pauly J, Nishimura D, Hu B, McConnell M (2004) Spiral magnetic resonance coronary angiography-direct comparison of 1.5 Tesla vs. 3 Tesla. J Cardiovasc Magn Reson 6:877-884

17. Sommer T, Hackenbroch M, Hofer U, Schmiedel A, Willinek WA, Flacke S, Gieseke J, Träber F, Fimmers R, Litt H, Schild H (2005) Coronary MR angiography at 3.0 T versus that at $1.5 \mathrm{~T}$ : initial results in patients suspected of having coronary artery disease. Radiology 234:718-725

18. Bi X, Deshpande V, Simonetti O, Laub G, Li D (2005) Three-dimensional breathhold SSFP coronary MRA: a comparison between 1.5T and 3.0T. J Magn Reson Imaging 22:206-212

19. Stuber M, Botnar RM, Fischer SE, Lamerichs R, Smink J, Harvey P, Manning WJ (2002) Preliminary report on in vivo coronary MRA at 3 Tesla in humans. Magn Reson Med 48:425-429

20. Nehrke K, Börnert P, Mazurkewitz P, Winkelmann R, Grässlin I (2006) Free-breathing whole-heart coronary MR angiography on a clinical scanner in four minutes. J Magn Reson Imaging 23:752-756

21. Niendorf T, Hardy CJ, Giaquinto RO, Gross P, Cline HE, Zhu Y, Kenwood G, Cohen S, Grant AK, Joshi S, Rofsky NM, Sodickson DK (2006) Toward single breath-hold whole-heart coverage coronary MRA using highly accelerated parallel imaging with a 32-channel MR system. Magn Reson Med 56:167-176

22. Niendorf T, Sodickson DK (2008) Highly accelerated cardiovascular MR imaging using many channel technology: concepts and clinical applications. Eur Radiol 18: 87-102

23. Meisel E, Pfeiffer D, Engelmann L, Tebbenjohanns J, Schubert B, Hahn S, Fleck E, Butter C (2001) Investigation of coronary venous anatomy by retrograde venography in patients with malignant ventricular tachycardia. Circulation 104:442-447 